\title{
Monotone paths in random hypergraphs
}

\author{
P. Majer*, M. Novaga ${ }^{\dagger}$ \\ Submitted: Nov 14, 2011; Accepted: Mar 29, 2012; Published: Apr 7, 2012
}

\begin{abstract}
We determine the probability thresholds for the existence of monotone paths, of finite and infinite length, in random oriented graphs with vertex set $\mathbb{N}^{[k]}$, the set of all increasing $k$-tuples in $\mathbb{N}$. These graphs appear as line graph of uniform hypergraphs with vertex set $\mathbb{N}$.
\end{abstract}

\section{Introduction}

In this paper we are interested in oriented graphs with vertex set $\mathbb{N}^{[k]}$, the set of all increasing $k$-tuples of elements of $\mathbb{N}$. We recall that an oriented graph $G$ is a pair of sets $\left(V_{G}, E_{G}\right)$, where $V_{G}$ is the set of vertices and $E_{G} \subseteq V_{G} \times V_{G}$ is the set of edges of $G$, such that $(a, b) \in E_{G}$ implies $(b, a) \notin E_{G}$ for all $a, b \in V_{G}$.

We point out that such graphs naturally arise as line graphs of $k$-uniform hypergraphs with vertex set $\mathbb{N}$ [VV:09]. Indeed, every $k$-edge of such a hypergraph is an element of $\mathbb{N}^{[k]}$, and we can uniquely associate to the hypergraph the line graph with vertex set $\mathbb{N}^{[k]}$, where two vertices are linked if the two corresponding hyperedges have intersection of a prescribed type.

A random subgraph of $G$ is a random choice of its vertices: more precisely, we associate to each vertex $v \in V_{G}=\mathbb{N}^{[k]}$ a measurable set $X_{v} \subseteq \Omega$, where $(\Omega, \mu)$ is a given probability space. We recall that $G$ can be equivalently defined by means of a $\mu$-measurable function $X: \Omega \rightarrow 2^{V_{G}}$, such that $X_{v}:=\{\omega \in \Omega: v \in X(\omega)\}$ for all $v \in V_{G}$.

Notice that we consider graphs with random vertices rather than random edges, as this class of graphs seems more natural for the questions we address in this paper. However, for the sake of completeness, in Section 4.5 we briefly discuss the case of random edges.

The main problem we study in this paper is whether a random subgraph $X(\omega)$ of $G$ contains an infinite path for some $\omega \in \Omega$.

*Dipartimento di Matematica, Università di Pisa, Largo B. Pontecorvo 5, 56127 Pisa, Italy, e-mail: majer@dm.unipi.it

${ }^{\dagger}$ Dipartimento di Matematica, Università di Padova, via Trieste 63, 35121 Padova, Italy, e-mail: novaga@dm.unipi.it 
Problem 1. For all $v \in V_{G}=\mathbb{N}^{[k]}$ let $X_{v}$ be a measurable subset of a probability space $(\Omega, \mu)$, with $\mu\left(X_{v}\right) \geqslant \lambda \in[0,1]$. We ask for which values of $\lambda$ there exists an infinite sequence of vertices $v_{i}$ of $G$ such that $\left(v_{i}, v_{i+1}\right)$ is an edge of $G$ for all $i \in \mathbb{N}$ and $\bigcap_{i \in \mathbb{N}} X_{v_{i}}$ is non-empty.

In order to answer to Problem 1, we have to compute the threshold

$$
\lambda_{G}:=\sup \left\{\inf _{v \in V_{G}=\mathbb{N}^{[k]}} \mu\left(X_{v}\right): X \text { random subgraph of } G \text { without infinite paths }\right\}
$$

and we also want to estimate the probability

$$
\mu(\{\omega \in \Omega: X(\omega) \text { contains an infinite path }\})
$$

that the random graph $X$ contains an infinite path, in terms of the parameter $\lambda$.

Problem 1 was first posed in [EH:64] for the graphs $G_{k}$ defined in Section 3.3, that is, $V_{G_{k}}=\mathbb{N}^{[k]}$ and $\left(v_{1}, v_{2}\right) \in E_{G_{k}}$, with $v_{1}=\left(i_{0}, \ldots, i_{k-1}\right)$ and $v_{2}=\left(j_{0}, \ldots, j_{k-1}\right)$, if and only if $j_{\ell}=i_{\ell+1}$ for all $\ell \in\{0, \ldots, k-2\}$. A complete solution of Problem 1 for $k=2$ ( $k=1$ being trivial) was given in [FT:85], where the authors show that an infinite path necessarily exists if $\lambda \geqslant 1 / 2$, while there are counterexamples for $\lambda<1 / 2$. In the paper [BMN:12] we present a different proof of this result, relying on the theory of exchangeable measures introduced by De Finetti in [DF:30] and later developed by many other authors (see [A:08] and references therein).

In this paper, following the approach of [BMN:12], we provide a solution to Problem 1 for a class of graphs which includes the graphs $G_{k}$ for all $k \in \mathbb{N}$ (see Theorem 4.5).

An important notion in this paper is the joint contractability of measures (see Section 3 and $[\mathrm{K}: 92, \mathrm{~K}: 05]$ ), which extends the notion of exchangeability by demanding a smaller class of symmetries. For such measures there is a remarkable representation theorem (Theorem 3.1) due to Kallenberg [K:92], which is crucial for the present work. Using this representation, we are able to reformulate Problem 1 as a variational problem on the unit cube, which we then solve by combinatorial methods (see Lemma 4.1).

As discussed in detail in Section 4.2, if a random subgraph $X$ of $G$ has no infinite paths, one can define a $\mu$-measurable map $\varphi: \Omega \rightarrow \omega_{1}^{\mathbb{N}^{[k]}}$ where $\varphi(\omega, v)$ is the rank of the vertex $v \in V_{G}=\mathbb{N}^{[k]}$ in the graph $X(\omega)$. It turns out that $\varphi_{*}(\mu)$ is a compactly supported Borel measure on $\omega_{1}^{\mathbb{N}}$, and that

$$
\varphi\left(X_{v}\right) \subseteq A_{v}:=\left\{x \in \omega_{1}^{\mathbb{N}^{[k]}}: x_{v}>x_{v^{\prime}} \text { for all } v^{\prime} \text { such that }\left(v, v^{\prime}\right) \in E_{G}\right\} .
$$

As a consequence, in the determination of the threshold $\lambda_{G}$ we can set $\Omega=\omega_{1}^{\mathbb{N}^{[k]}}$ and $X_{v}=A_{v}$, and reduce to a variational problem on the set $\mathcal{M}_{c}^{1}\left(\omega_{1}^{\mathbb{N}^{[k]}}\right)$ of all compactly supported probability measures on $\omega_{1}^{\mathbb{N}^{[k]}}$, namely

$$
\lambda_{G}=\sup _{m \in \mathcal{M}_{c}^{1}\left(\omega_{1}^{\mathbb{N}}[k]\right.} \inf _{v \in \mathbb{N}^{[k]}} m\left(A_{v}\right) .
$$


As a next step, we show that in (1.2) we can equivalently take the supremum in the smaller class of the compactly supported contractible measures on $\omega_{1}^{\mathbb{N}^{[k]}}$. Thanks to this reduction, we can explicitly compute $\lambda_{G}$ when $G$ is a simple contractible graph (Theorem 4.5).

A closely related problem, which we discuss at the end of the paper, is finding the thresholds for paths of finite length. We observe that this type of questions are reminiscent of Turán's problem [DC:83], and the answer is expected to be difficult in general. We mention that the case of paths of length 2 in the graphs $G_{k}$ mentioned above has been already considered in [TW:98], where the authors determine the precise thresholds for $k \in\{2,3\}$, show upper and lower bounds for all $k \in \mathbb{N}$, and make a conjecture for $k \in\{4,5\}$. In Section 4.4, we determine the thresholds for all $k$ odd, thus confirming the conjecture made in [TW:98] for $k=5$, while the case of $k$ even remains open.

\section{Notation}

Given $p \in \mathbb{N}$, we identify $p$ with the set $\{0, \ldots, p-1\}$. Given a topological space $S$ and $k \in \mathbb{N}$, we let $S^{[k]}$ be the set of all subsets of $S$ of cardinality $k$, endowed with the product topology, and we let $S^{[\leqslant k]}$ be the set of all subsets of $S$ of cardinality at most $k$. Notice that, if $S$ has cardinality $k \in \mathbb{N}$, then $S^{[\leqslant k]}=S^{[\leqslant j]}$ for all $j>k$ and $S^{[\leqslant k]}=\mathcal{P}(S)$ is the power set of $S$. If $S$ is ordered, we can identify $S^{[k]}$ with the set of $k$-tuples $\left(i_{0}, \ldots, i_{k-1}\right)$ with $i_{0}<\cdots<i_{k-1}$.

Given a compact metric space $\Lambda$, we let $\Lambda^{\mathbb{N}^{[k]}}$ be the space of all sequences with indices in $\mathbb{N}^{[k]}$ and values in $\Lambda$, endowed with the product topology, and $\mathcal{M}\left(\Lambda^{\mathbb{N}[k]}\right)$ be the space of all Radon measures on $\Lambda^{\mathbb{N}^{[k]}}$, endowed with the weak* topology. The subset $\mathcal{M}_{1}\left(\Lambda^{\mathbb{N}^{[k]}}\right) \subset \mathcal{M}\left(\Lambda^{\mathbb{N}^{[k]}}\right)$ denotes the (compact metrizable) space of all probability measures on $\Lambda^{\mathbb{N}^{[k]}}$.

We denote by $\omega_{1}$ the first uncountable ordinal and by $\mathcal{M}_{\mathrm{c}}\left(\omega_{1}^{\mathbb{N}^{[k]}}\right)$ the set of all probability measures on $\omega_{1}^{\mathbb{N}^{[k]}}$ with compact support, that is, with support in $\alpha^{\mathbb{N}^{[k]}}$ for some $\alpha<\omega_{1}$.

We let $\operatorname{Incr}(\mathbb{N}) \subset \mathbb{N}^{\mathbb{N}}$ be the family of all maps $\sigma: \mathbb{N} \rightarrow \mathbb{N}$ which are strictly increasing, and we let $s: \mathbb{N} \rightarrow \mathbb{N}$ be the shift map defined as $s(i)=i+1$ for all $i \in \mathbb{N}$.

As a general rule, given a map $f$ we use the symbol $f_{*}\left(\right.$ resp. $\left.f^{*}\right)$ to indicate a covariant (resp. contravariant) map induced by $f$. Due to possible iteration of the previous rule, we still use the symbol $*$ to sum-up a certain number of $*$ 's, keeping the previous convention in order to distinguish a covariant from a contravariant action. In particular, given a map $\sigma: \mathbb{N} \rightarrow \mathbb{N}$ we let $\sigma_{*}: \mathbb{N}^{[k]} \rightarrow \mathbb{N}^{[k]}$ be defined as $\sigma_{*}\left(i_{0}, \ldots, i_{k-1}\right)=\left(\sigma\left(i_{0}\right), \ldots, \sigma\left(i_{k-1}\right)\right)$, we let $\sigma^{*}: \Lambda^{\mathbb{N}^{[k]}} \rightarrow \Lambda^{\mathbb{N}^{[k]}}$ be defined as $\sigma^{*}(x)_{i}=x_{\sigma_{*}(i)}$ for all $i \in \mathbb{N}^{[k]}$, and we let $\sigma^{*}: \mathcal{M}\left(\Lambda^{\mathbb{N}^{[k]}}\right) \rightarrow \mathcal{M}\left(\Lambda^{\mathbb{N}^{[k]}}\right)$ be the corresponding pushforward map. 


\section{Contractible measures and graphs}

\subsection{Contractible measures}

Let $k \in \mathbb{N}$, let $\Lambda$ be a compact metric space, and let $m$ be a Borel probability measure on $\Lambda^{\mathbb{N}^{[k]}}$. We say that $m$ is (jointly) contractible if $m=\sigma^{*}(m)$ for all $\sigma \in \operatorname{Incr}(\mathbb{N})$.

Noice that, when $k=1$, the notion of contractible measure reduces to the well-known notion of exchangeable measure, introduced by De Finetti in [DF:30].

Given $k \in \mathbb{N}$ and $f:[0,1]^{k^{[\leqslant k]}} \rightarrow \Lambda$, we let $c: \mathbb{N}^{[k]} \times k^{[\leqslant k]} \rightarrow \mathbb{N}^{[\leqslant k]}$ be the composition function, and $\hat{f}:[0,1]^{\mathbb{N}[\leqslant k]} \rightarrow \Lambda^{\mathbb{N}^{[k]}}$ be defined as the composition of $f_{*}$ and $c^{*}$, that is

$$
\hat{f}=f_{*} \circ c^{*},
$$

where $f_{*}:\left([0,1]^{k^{[k k]}}\right)^{\mathbb{N}^{[k]}} \rightarrow \Lambda^{\mathbb{N}^{[k]}}, c^{*}:[0,1]^{\mathbb{N}^{[\leqslant k]}} \rightarrow[0,1]^{\mathbb{N}^{[k]} \times k^{[\leqslant k]}}$. Notice that $\hat{f}$ induces a $\operatorname{map} \hat{f}_{*}: \mathcal{M}_{1}\left([0,1]^{\mathbb{N}[\leqslant k]}\right) \rightarrow \mathcal{M}_{1}\left(\Lambda^{\mathbb{N}[k]}\right)$.

Contractible measures admit the following representation [K:05, Theorem 7.15]:

Theorem 3.1. Let $\Lambda$ be a compact subset of $[0,1]$ and let $m$ be a contractible measure on $\Lambda^{\mathbb{N}^{[k]}}$. Then, there exists a measurable function $f:[0,1]^{k^{[\leqslant k]}} \rightarrow \Lambda$ such that $m=\hat{f}_{*}(\mathcal{L})$, where $\mathcal{L}$ is the product Lebesgue measure on $[0,1]^{\mathbb{N}[\leqslant k]}$.

We observe that Theorem 3.1, in the particular case $k=1$, implies the representation result for exchangeable measures due to De Finetti [DF:30] (see also [HS:55]).

\section{$3.2 \quad$ Asymptotically contractible measures}

We say that a measure $m \in \mathcal{M}_{1}\left(\Lambda^{\mathbb{N}^{[k]}}\right)$ is asymptotically contractible if there exists a contractible measure $m^{\prime}$ such that there exists the limit

$$
m^{\prime}=\lim _{\substack{\min \theta \rightarrow \infty \\ \theta \in \operatorname{Incr}(\mathbb{N})}} \theta^{*}(m)
$$

The following result can be proved as in [BMN:12, Theorem B.8].

Proposition 3.2. Given $m \in \mathcal{M}_{1}\left(\Lambda^{\mathbb{N}^{[k]}}\right)$ there exists $\sigma \in \operatorname{Incr}(\mathbb{N})$ such that the measure $\sigma^{*}(m)$ is asymptotically contractible.

Proof. Fix $m \in \mathcal{M}_{1}\left(\Lambda^{\mathbb{N}[k]}\right)$. Given $r \in \mathbb{N}$ consider the function $f: \mathbb{N}^{[r]} \rightarrow \mathcal{M}_{1}\left(\Lambda^{\left[{ }^{[k]}\right.}\right)$ sending $\iota \in \mathbb{N}^{[r]}$ to $\iota^{*}(m) \in \mathcal{M}_{1}\left(\Lambda^{r^{[k]}}\right)$. By Lemma 3.3 below, applied with $M=\mathcal{M}_{1}\left(\Lambda^{r^{[k]}}\right)$, there is an infinite set $J_{r} \subset \mathbb{N}$ such that

$$
\lim _{\substack{\min (\iota) \rightarrow \infty \\ \iota \in J_{r}^{[r]}}} \iota^{*}(m)
$$

exists in $\mathcal{M}^{1}\left(\Lambda^{r^{[k]}}\right)$. By a diagonal argument we choose the same set $J=J_{r}$ for all $r$. Letting $\sigma \in \operatorname{Incr}(\mathbb{N})$ be such that $\sigma(\mathbb{N})=J$, we claim that $\sigma^{*}(m)$ is asymptotically 
exchangeable. To this aim consider $m_{k}:=\left(s^{*}\right)^{k} \sigma^{*}(m) \in \mathcal{M}_{1}\left(\Lambda^{\mathbb{N}^{[k]}}\right)$. By compactness there exists an accumulation point $m^{\prime} \in \mathcal{M}_{1}\left(\Lambda^{\mathbb{N}^{[k]}}\right)$ of the sequence $\left\{m_{k}\right\}_{k \in \mathbb{N}}$, and we claim that

$$
\lim _{\substack{\min (\theta) \rightarrow \infty \\ \theta \in \operatorname{Incr}(\mathbb{N})}} \theta^{*} \sigma^{*}(m)=m^{\prime}
$$

Notice that the claim also implies that $m^{\prime}$ is contractible. Indeed, given an increasing function $\gamma: \mathbb{N} \rightarrow \mathbb{N}$, to show $\gamma^{*}\left(m^{\prime}\right)=m^{\prime}$ it is enough to replace $\theta$ with $\theta \circ \gamma$ in (3.2). Since the subset of $C\left(\Lambda^{\mathbb{N}^{[k]}}\right)$ consising of the functions depending on finitely many coordinates is dense in $C\left(\Lambda^{\mathbb{N}}[k]\right)$, it suffices to prove that the limit

$$
\lim _{\substack{\min (\theta) \rightarrow \infty \\ \theta \in \operatorname{Incr}(\mathbb{N})}} \iota^{*} \theta^{*} \sigma^{*}(m)
$$

exists in $\mathcal{M}_{1}\left(\Lambda^{[k]}\right)$ for all $r \in \mathbb{N}$ and $\iota \in \mathbb{N}^{[r]}$, but this is just a special case of (3.1).

We conclude this section by stating a topological version of Ramsey Theorem [BMN:12, Lemma A.1].

Lemma 3.3. Let $M$ be a compact metric space, let $k \in \mathbb{N}$, and let $f: \mathbb{N}^{[k]} \rightarrow M$. Then there exists an infinite set $J \subset \mathbb{N}$ such that there exists the limit

$$
\lim _{\substack{\left(i_{1}, \ldots, i_{k}\right) \rightarrow+\infty \\\left(i_{1}, \ldots, i_{k}\right) \in J^{[k]}}} f\left(i_{1}, \ldots, i_{k}\right) .
$$

\subsection{Contractible graphs}

We now introduce the class of ambient graphs in which we consider Problem 1.

Definition 3.4. Let $G$ be an oriented graph with $V_{G}=\mathbb{N}^{[k]}$ for some $k \in \mathbb{N}$. We say that $G$ is contractible if

$-(a, b) \in E_{G} \Rightarrow a_{i}<b_{i}$ for all $0 \leqslant i \leqslant k-1$;

$-(a, b) \in E_{G} \Rightarrow\left(\varphi_{*}(a), \varphi_{*}(b)\right) \in E_{G}$ for all $\varphi \in \operatorname{Incr}(\mathbb{N})$.

We say that a map $\tau: S_{\tau} \rightarrow \mathbb{N}$ is an order relation of length $k \in \mathbb{N}$ if

$-S_{\tau}=\left\{a_{0}, \ldots, a_{k-1}\right\} \subset \mathbb{N}$, with $a_{0}<\cdots<a_{k-1}$;

$-a_{i}<\tau\left(a_{i}\right)$ for all $0 \leqslant i \leqslant k-1$;

$-\tau\left(a_{i}\right)<\tau\left(a_{i+1}\right)$ for all $0 \leqslant i \leqslant k-2$. 
We say that two order relations $\tau, \tau^{\prime}$ are equivalent if there exist $\sigma, \sigma^{\prime} \in \operatorname{Incr}(\mathbb{N})$ such that

$$
\sigma\left(a_{i}\right)=\sigma^{\prime}\left(a_{i}^{\prime}\right) \text { and } \sigma \circ \tau\left(a_{i}\right)=\sigma^{\prime} \circ \tau^{\prime}\left(a_{i}^{\prime}\right) \text { for all } 0 \leqslant i \leqslant k-1 .
$$

We say that the couple $\left(v, v^{\prime}\right) \in \mathbb{N}^{[k]} \times \mathbb{N}^{[k]}$ satisfies the order relation $\tau$ if there exists $\sigma \in \operatorname{Incr}(\mathbb{N})$ such that $v_{i}=\sigma\left(a_{i}\right)$ and $v_{i}^{\prime}=\sigma \circ \tau\left(a_{i}\right)$ for all $0 \leqslant i \leqslant k-1$. Notice that $\tau$ can be naturally extended to a function $\tau_{*}: S_{\tau}^{[\leqslant k]} \rightarrow \mathbb{N}^{[\leqslant k]}$ defined on all the $r$-tuples of elements in $S_{\tau}$. Given an order relation $\tau$, we let

$$
\Sigma_{\tau}:=\left\{a_{i} \in S_{\tau}: i \leqslant k-1, \tau\left(a_{i}\right)=a_{j} \text { for some } 0 \leqslant j \leqslant k-1\right\} \subseteq S_{\tau} .
$$

We denote by $\mathcal{E}_{k}$ the set of all order relations of length $k$. Given $\mathcal{C} \subseteq \mathcal{E}_{k}$ we let $G_{\mathcal{C}}$ be the graph with vertex set $\mathbb{N}^{[k]}$ and such that $\left(v, v^{\prime}\right)$ is an edge of $G_{\mathcal{C}}$ if and only if $\left(v, v^{\prime}\right)$ satisfies $\tau$ for some $\tau \in \mathcal{C}$. Notice that $G_{\mathcal{C}}$ is a contractible graph for all $\mathcal{C} \subseteq \mathcal{E}_{k}$.

Definition 3.5. We say that a contractible graph $G$ is simple if $G=G_{\tau}$ for some $\tau \in \mathcal{E}_{k}$.

A relevant example of such graphs is the graph $G_{k}=\left(V_{G_{k}}, E_{G_{k}}\right)$ with vertices $V_{G_{k}}=$ $\mathbb{N}^{[k]}$ and such that $\left(v, v^{\prime}\right) \in E_{G_{k}}$ iff $v_{i}^{\prime}=v_{i+1}$ for all $i \in\{0, \ldots, k-2\}$.

\section{Probability thresholds}

In this section we give an answer to Problem 1 under the assumption that $G$ is a simple contractible graph.

\subsection{A variational problem in the unit cube}

We first discuss a variational problem on the unit cube, which is related to Problem 1. From now on, given a set $A \subseteq[0,1]^{S}$ where $S$ is a countable set, we set $|A|:=\mathcal{L}(A)$ where $\mathcal{L}$ is the product Lebesgue measure on $[0,1]^{S}$.

Lemma 4.1. Let $S$ be a set, let $S_{0} \subset S$ be a finite subset, and let $\tau: S_{0} \rightarrow S$, so that $\tau^{*}$ maps $[0,1]^{S}$ into $[0,1]^{S_{0}}$. For all measurable functions $f:[0,1]^{S_{0}} \rightarrow[0,1]$ we let

$$
Z_{\tau}(f):=\left\{x \in[0,1]^{S}: f\left(\left.x\right|_{S_{0}}\right)>f\left(\tau^{*}(x)\right)\right\} .
$$

If $\tau$ has no cycles, we have

$$
\sup _{f:[0,1]^{S_{0} \rightarrow[0,1]}}\left|Z_{\tau}(f)\right|=1-\frac{1}{w(\tau)}
$$

where

$$
w(\tau):=1+\max \left\{n \in \mathbb{N}: \exists s \in S_{0} \text { s.t. } \tau^{j}(s) \in S_{0} \forall 1 \leqslant j \leqslant n-1\right\} \geqslant 2 .
$$


Proof. Assume first that $\tau$ can be extended to a $p$-periodic function $\tilde{\tau}$ on the whole of $S$, for some $p \in \mathbb{N}$. Let $f:[0,1]^{S_{0}} \rightarrow[0,1]$ and notice that

$$
\bigcap_{j=0}^{p-1}\left(\tilde{\tau}^{*}\right)^{j}\left(Z_{\tau}(f)\right)=\emptyset
$$

It then follows

$$
p\left(1-\left|Z_{\tau}(f)\right|\right)=p\left|Z_{\tau}(f)^{c}\right| \geqslant\left|\bigcup_{j=0}^{p-1}\left(\left(\tilde{\tau}^{*}\right)^{j}\left(Z_{\tau}(f)\right)\right)^{c}\right|=1-\left|\bigcap_{j=0}^{p-1}\left(\tilde{\tau}^{*}\right)^{j}\left(Z_{\tau}(f)\right)\right|=1,
$$

which implies

$$
\left|Z_{\tau}(f)\right| \leqslant 1-\frac{1}{p}
$$

Observe that, by definition of $w(\tau)$, we can find a $w(\tau)$-periodic function $\tilde{\tau}: S \rightarrow S$ such that $\left.\tilde{\tau}\right|_{S_{0}}=\left.\tau\right|_{S_{0}}$. From (4.3) it then follows

$$
\left|Z_{\tau}(f)\right| \leqslant 1-\frac{1}{w(\tau)}
$$

We now prove the opposite inequality. By definition of $w(\tau)$ there exists $\bar{s} \in S_{0}$ such that $\tau^{j}(\bar{s}) \in S_{0}$ for all $1 \leqslant j \leqslant w(\tau)-2$. Let us consider the function

$$
f_{\varepsilon}(x)=\frac{1}{1+\varepsilon w(\tau)}\left(\max _{0 \leqslant j \leqslant w(\tau)-2} x_{\tau^{j}(\bar{s})}+\varepsilon \bar{j}\right),
$$

where $1 /(1+\varepsilon w(\tau))$ is just a normalization factor in order to have $f_{\varepsilon}(x) \in[0,1]$, and $\bar{j}=\bar{j}(x) \in \mathbb{N}$ is such that $x_{\tau^{\bar{j}}(\bar{s})}=\max _{0 \leqslant j \leqslant w(\tau)-2} x_{\tau^{j}(\bar{s})}$. Notice that $\bar{j}(x)$ is well-defined out of a set of zero measure. We then have

$$
\left|Z_{\tau}\left(f_{\varepsilon}\right)\right| \geqslant \lim _{\varepsilon \rightarrow 0}\left|Z_{\tau}\left(f_{\varepsilon}\right)\right|=1-\frac{1}{w(\tau)}
$$

which gives (4.2).

Notice that $w(\tau)$ depends only only on $\left(\tau, S_{0}\right)$ and is independent of $S$, so that for instance one can substitute $S$ with the finite set $S_{0} \cup \tau\left(S_{0}\right)$.

Example 4.2. In the particular case where $S=\mathbb{N}, S_{0}=\{1, \ldots, k\}$ and $\tau$ is the shift map restricted to $S_{0}$, i.e. $\tau(i)=i+1$ we have $w(\tau)=k+1$, so that

$$
\sup _{f:[0,1]^{S_{0} \rightarrow I}}\left|Z_{\tau}(f)\right|=1-\frac{1}{k+1} .
$$




\subsection{A canonical probability space}

Following [BMN:12], we reformulate Problem 1 as a variational problem on a suitable space of sequences. For all $\omega \in \Omega$, we consider the oriented graph $X(\omega)<G$ whose vertices are all $v \in V_{G}$ such that $\omega \in X_{v}$. Given $v \in \mathbb{N}^{[k]}$, we also let $Y_{v} \subseteq X_{v}$ be the subset of all $\omega$ such that $X(\omega)$ contains an infinite path starting from $v$, i.e. there exists an infinite sequence $\left\{v_{k}\right\}_{k \in \mathbb{N}}$, with $v_{1}=v$ and $\omega \in \bigcap_{k} X_{v_{k}}$.

We recall that a partially ordered set admits a decreasing function into the first uncountable ordinal $\omega_{1}$ (called height or rank function) if and only if it has no infinite increasing sequences, we can define a measurable map $\varphi: \Omega \times \mathbb{N}^{[k]} \rightarrow \omega_{1}+1$ by setting

$$
\varphi(\omega, v)= \begin{cases}\sup _{\left\{v^{\prime} \in \mathbb{N}^{[k]}:\left(v, v^{\prime}\right) \in E_{G}, \omega \in X_{v^{\prime}}\right\}} \varphi\left(\omega, v^{\prime}\right)+1 & \text { if } \omega \in X_{v} \backslash Y_{v}, \\ 0 & \text { if } \omega \notin X_{v} \\ \omega_{1} & \text { if } \omega \in Y_{v} .\end{cases}
$$

For simplicity of notation, we still denote by $\varphi$ the map $\varphi: \Omega \rightarrow\left(\omega_{1}+1\right)^{\mathbb{N}^{[k]}}$ defined as $\varphi(\omega)_{v}=\varphi(\omega, v)$.

Remark 4.3. We have $\varphi(\omega, v)=\varphi_{\omega_{1}}(\omega, v)$ where $\varphi_{\alpha}: \Omega \rightarrow\left(\omega_{1}+1\right)^{\mathbb{N}^{[k]}}$ is the truncation $\varphi_{\alpha}:=\min (\varphi, \alpha)$, that we can equivalently define by induction on $\alpha \leqslant \omega_{1}$ as

$$
\begin{aligned}
& \varphi_{0}(\omega, v)=0 \\
& \varphi_{\alpha}(\omega, v)=\sup \left\{\varphi_{\beta}\left(\omega, v^{\prime}\right)+1: \beta<\alpha,\left(v, v^{\prime}\right) \in E_{G}, \omega \in X_{v^{\prime}}\right\} .
\end{aligned}
$$

Notice that $\varphi(\omega, v)<\omega_{1}$ iff there is no infinite path in $X(\omega)$ starting from $v$, that is $\omega \notin Y_{v}$, and in this case $\varphi(\omega, v)$ is precisely the height of the vertex $v$ in $X(\omega)$. Indeed, if $\omega \in Y_{v}$ then $\varphi(\omega, v)=\omega_{1}$ by definition. On the other hand, if $\varphi(\omega, v)=\omega_{1}$ then there exists $v^{\prime}$ such that $\left(v, v^{\prime}\right) \in E_{G}, \omega \in X_{v^{\prime}}$ and $\varphi\left(\omega, v^{\prime}\right)=\omega_{1}$, since otherwise $\varphi(\omega, v)$ would also be less than $\omega_{1}$, being the supremum of a countable set of countable ordinals. By iteration we then get $\omega \in Y_{v}$. In particular, if $X$ has no infinite paths, then the function $\varphi$ takes value in $\omega_{1}^{\mathbb{N}^{[k]}}$ and, if there are no paths of length $p$, then it takes values in $p^{\mathbb{N}^{[k]}}$.

Lemma 4.4. The following assertions hold:

1. the set $P:=\{\omega \in \Omega: X(\omega)$ has an infinite path $\}$ is $\mu$-measurable;

2. for all $\alpha \leqslant \omega_{1}$ and $v \in V_{G}$, the set $\{\omega \in \Omega: \varphi(\omega, v)=\alpha\}$ is $\mu$-measurable;

3. $\varphi: \Omega \rightarrow\left(\omega_{1}+1\right)^{V_{G}}$ is $\mu$-measurable and its restriction to $\Omega \backslash P$ is essentially bounded.

Proof. Since taking the supremum over a countable set preserves measurability, it follows that the sets $\{\omega \in \Omega: \varphi(\omega, v)=\alpha\}$ are measurable for all $v \in V_{G}$ and $\alpha<\omega_{1}$. We will show that $\left\{\omega \in \Omega: \varphi(\omega, v)=\omega_{1}\right\}$ is $\mu$-measurable, namely it is the union of a measurable set and a $\mu$-null set. Fix $v \in V_{G}$. The sequence of values $\mu(\{\omega \in \Omega: \varphi(\omega, v) \leqslant \beta\})$ is increasing with respect to the countable ordinal $\beta$ and uniformly bounded by $1=\mu(\Omega)$, therefore it is stationary at some finite value. So there is $\alpha_{0}<\omega_{1}$ such that

$$
\mu(\{\omega \in \Omega: \varphi(\omega, v)=\beta\})=0 \quad \text { for } \alpha_{0} \leqslant \beta<\omega_{1} .
$$


It follows that $\left\{\omega \in \Omega: \varphi(\omega, v)=\omega_{1}\right\}$ is $\mu$-measurable and $\varphi$ is $\mu$-measurable. Since $P=\cup_{v}\left\{\omega \in \Omega: \varphi(\omega, v)=\omega_{1}\right\}$, we have that $P$ is $\mu$-measurable, too.

As a consequence, if $X$ has no infinite paths, then the function $\varphi$ maps $\Omega$ into the Cantor space $\alpha^{\mathbb{N}^{[k]}}$ for some $\alpha<\omega_{1}$ (up to a set of zero measure), so that it induces a probability measure $m=\varphi_{*}(\mu)$ on $\omega_{1}^{\mathbb{N}^{[k]}}$ concentrated on $\alpha^{\mathbb{N}^{[k]}}$, i.e. $m\left(\alpha^{\mathbb{N}^{[k]}}\right)=1$. Moreover, for all $v \in V_{G}$ we have

$$
\varphi\left(X_{v}\right) \subseteq A_{v}:=\left\{x \in \omega_{1}^{\mathbb{N}^{[k]}}: x_{v}>x_{v^{\prime}} \text { for all } v^{\prime} \text { such that }\left(v, v^{\prime}\right) \in E_{G}\right\} .
$$

It then follows

$$
\lambda_{G}=\sup _{m \in \mathcal{M}_{\mathrm{c}}\left(\omega_{1}^{\mathbb{N}}[k]\right.} \inf _{v \in \mathbb{N}^{[k]}} m\left(A_{v}\right)
$$

\subsection{Infinite paths.}

We are ready to state the main result of this paper.

Theorem 4.5. Let $G=G_{\tau}$ be a simple contractible graph with $V_{G}=\mathbb{N}^{[k]}$. Then

$$
\lambda_{G}=1-\frac{1}{w\left(\left.\tau\right|_{\Sigma_{\tau}}\right)} .
$$

In particular, letting $X: \Omega \rightarrow 2^{V_{G}}$, the random graph $X$ has an infinite path if

$$
\lambda:=\inf _{v \in V_{G}} \mu\left(X_{v}\right)>\lambda_{G} .
$$

On the contrary, if $\lambda<\lambda_{G}$ we can find $X$ such that $X(\omega)$ has no infinite paths for some $\omega \in \Omega$.

Proof. We divide the proof into several steps.

Step 1. Recalling (4.5), we want to compute the supremum

$$
\sup _{m \in \mathcal{M}_{\mathrm{c}}\left(\omega_{1}^{\mathbb{N}}[k]\right)} \inf _{v \in \mathbb{N}^{[k]}} m\left(A_{v}\right)
$$

Since the support of $m$ is contained in $\alpha_{0}^{\mathbb{N}[k]}$, for some compact countable ordinal $\alpha_{0}$, thanks to Proposition 3.2 we can assume that $m$ is asymptotically contractible, so that in particular the sequence $m_{k}=\left(s^{*}\right)^{k}(m)$ converges to a contractible measure $m^{\prime} \in \mathcal{M}_{1}\left(\alpha_{0}^{\mathbb{N}^{[k]}}\right)$ in the weak* topology of $\mathcal{M}_{1}\left(\alpha_{0}^{\mathbb{N}[k]}\right)$. Moreover, one can prove by ordinal induction as in [BMN:12, Lemma 4.4] that for all $\alpha<\omega_{1}$ there holds

$$
\inf _{v \in \mathbb{N}^{[k]}} m\left(\left\{x \in A_{v}: x_{v} \leqslant \alpha\right\}\right) \leqslant \inf _{v \in \mathbb{N}^{[k]}} m^{\prime}\left(\left\{x \in A_{v}: x_{v} \leqslant \alpha\right\}\right)
$$

Therefore, we can assume that the measure $m$ in (4.5) is contractible. 
Step 2. Recalling the definition of the set $A_{v}$ in (4.4) and letting $S=S_{\tau}$ be the domain of $\tau$, by Theorem 3.1 we have

$$
\begin{aligned}
& \sup _{m \in \mathcal{M}_{\mathrm{c}}\left(\omega_{1}^{\mathbb{N}[k]}\right)} \inf _{v \in \mathbb{N}^{[k]}} m\left(A_{v}\right)=\sup _{\substack{m \in \mathcal{M}_{\mathrm{c}}\left(\omega_{1}^{\mathbb{N}[k]}\right) \\
m \text { contractible }}} \inf _{v \in \mathbb{N}^{[k]}} m\left(A_{v}\right)=\sup _{\substack{m \in \mathcal{M}_{\mathrm{c}}\left(\omega_{1}^{\mathbb{N}} \\
m\right. \text { contractible }}} m\left(A_{S}\right) \\
& =\sup _{\substack{f:[0,1]^{S}[\leqslant k] \rightarrow \alpha_{0} \\
\alpha_{0}<\omega_{1}}} \mid\left\{x \in[0,1]^{\mathbb{N}[\leqslant k]}: f\left(\left.x\right|_{S[\leqslant k]}\right)>f\left(\tau^{*} \sigma^{*}(x)\right) \forall \sigma \in \operatorname{Incr}(\mathbb{N}) \text { s.t. }\left.\sigma\right|_{S}=\mathrm{id}\right\} \mid \\
& \leqslant \sup _{f:[0,1]^{S}[\leqslant k] \rightarrow[0,1]} \mid\left\{x \in[0,1]^{\mathbb{N}[\leqslant k]}: f\left(\left.x\right|_{S[\leqslant k]}\right)>f\left(\tau^{*} \sigma^{*}(x)\right) \forall \sigma \in \operatorname{Incr}(\mathbb{N}) \text { s.t. }\left.\sigma\right|_{S}=\mathrm{id}\right\} \mid
\end{aligned}
$$

where the last inequality follows since every countable ordinal $\alpha_{0}$ can be embedded in $[0,1]$ with the induced ordering. We will show below that this inequality is indeed an equality.

Step 3. Recalling the definition of $\Sigma_{\tau}$ in (3.4), we observe that every $x \in[0,1]^{\mathbb{N} \leqslant k]}$ can be uniquely written as $x=(y, z)$, with $y=\left.x\right|_{\left.S^{[} \leqslant k\right]}$ and $z=\left.x\right|_{\left.\mathbb{N}^{[} \leqslant k\right]} \backslash S[\leqslant k]$. Similarly, we sometimes write $y \in[0,1]^{S^{[\leqslant k]}}$ as $y=\left(y^{\prime}, t\right)$, with $y^{\prime}=\left.y\right|_{\Sigma_{\tau}^{[\leqslant k]}}$ and $z=\left.x\right|_{S[\leqslant k] \backslash \Sigma_{\tau}^{[\leqslant k]}}$. For simplicity of notation, we let in the following $\tau_{0}: \Sigma_{\tau} \rightarrow S$ and $\tau_{1}: S \backslash \Sigma_{\tau} \rightarrow \mathbb{N} \backslash S$ be the restrictions of $\tau$ to $\Sigma_{\tau}$ and $S \backslash \Sigma_{\tau}$ respectively, so that $\tau_{0}^{*}:[0,1]^{S^{[\leqslant k]}} \rightarrow[0,1]^{\Sigma_{\tau}^{[\leqslant k]}}$. We will show that

$$
\begin{aligned}
& \mid\left\{x \in[0,1]^{\mathbb{N}[\leqslant k]}: f\left(\left.x\right|_{S[\leqslant k]}\right)>f\left(\tau^{*} \sigma^{*}(x)\right) \forall \sigma \in \operatorname{Incr}(\mathbb{N}) \text { s.t. }\left.\sigma\right|_{S}=\operatorname{id}\right\} \mid \\
& =\left|\left\{y \in[0,1]^{S^{[\leqslant k]}}: f(y)>f\left(\tau_{0}^{*}(y), t\right) \widetilde{\forall} t \in[0,1]^{S^{[\leqslant k]} \backslash \Sigma_{\tau}^{[\leqslant k]}}\right\}\right|
\end{aligned}
$$

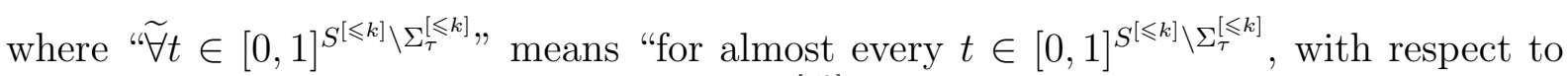
the product Lebesgue measure on $[0,1]^{S \leqslant k]} \backslash \Sigma_{\tau}^{[\leqslant k]}$ ". Indeed, for all $y \in S^{[\leqslant k]}$ we set

$$
J(y)=\left\{t \in[0,1]^{S[\leqslant k]} \backslash \Sigma_{\tau}^{[\leqslant k]}: f(y)>f\left(\tau_{0}^{*}(y), t\right)\right\} .
$$

Then, letting

$$
\mathcal{S}=\left\{\sigma \in \operatorname{Incr}(\mathbb{N}):\left.\sigma\right|_{S}=\mathrm{id}\right\},
$$

by Fubini-Tonelli Theorem we have

$$
\begin{aligned}
& \left|\left\{x \in[0,1]^{\mathbb{N}[\leqslant k]}: f\left(\left.x\right|_{S^{[\leqslant k]}}\right)>f\left(\tau^{*} \sigma^{*}(x)\right) \forall \sigma \in \mathcal{S}\right\}\right| \\
& =\left|\left\{x=(y, z) \in[0,1]^{\mathbb{N}[\leqslant k]}: f(y)>f\left(\tau_{0}^{*}(y), \tau_{1}^{*} \sigma_{1}^{*}(z)\right) \forall \sigma \in \mathcal{S}\right\}\right| \\
& =\int_{[0,1]^{S[\leqslant k]}}\left|\left\{z \in[0,1]^{\mathbb{N}[\leqslant k]} \backslash S^{[\leqslant k]}: f(y)>f\left(\tau_{0}^{*}(y), \tau_{1}^{*} \sigma_{1}^{*}(z)\right) \forall \sigma \in \mathcal{S}\right\}\right| d y \\
& =\int_{[0,1]^{S}[\leqslant k]}\left|\left\{z \in[0,1]^{\mathbb{N}^{[\leqslant k]} \backslash S^{[\leqslant k]}}: \tau_{1}^{*} \sigma_{1}^{*}(z) \in J(y) \forall \sigma \in \mathcal{S}\right\}\right| d y
\end{aligned}
$$


where we denoted by $\sigma_{1}: \mathbb{N} \backslash S \rightarrow \mathbb{N} \backslash S$ the restriction of $\sigma$ to $\mathbb{N} \backslash S$. Notice that the set

$$
\left\{z \in[0,1]^{\mathbb{N}[\leqslant k]} \backslash S^{[\leqslant k]}: \tau_{1}^{*} \sigma_{1}^{*}(z) \in J(y) \forall \sigma \in \mathcal{S}\right\}
$$

appearing in the last term of (4.9), is contained in an infinite product of the set $J(y)$, therefore its measure is nonzero if and only if $|J(y)|=1$. From (4.9) it then follows

$$
\begin{aligned}
& \left|\left\{x \in[0,1]^{\mathbb{N}[\leqslant k]}: f\left(\left.x\right|_{S[\leqslant k]}\right)>f\left(\tau^{*} \sigma^{*}(x)\right) \forall \sigma \in \mathcal{S}\right\}\right| \\
& =\int_{[0,1]^{S}[\leqslant k]}\lfloor|J(y)|\rfloor d y \\
& =\left|\left\{y \in[0,1]^{S^{[\leqslant k]}}:|J(y)|=1\right\}\right| \\
& =\left|\left\{y \in[0,1]^{S^{[\leqslant k]}}: f(y)>f\left(\tau_{0}^{*}(y), t\right) \widetilde{\forall} t \in[0,1]^{S^{[\leqslant k]} \backslash \Sigma_{\tau}^{[\leqslant k]}}\right\}\right|
\end{aligned}
$$

which proves (4.8).

Step 4. Given $f:[0,1]^{S_{[\leqslant k]}} \rightarrow[0,1]$ and $\varepsilon>0$, we define $g_{f, \varepsilon}: \Sigma_{\tau}^{[\leqslant k]} \rightarrow[0,1]$ as

$$
g_{f, \varepsilon}\left(y^{\prime}\right):=\sup \left\{\lambda \in[0,1]:\left|\left\{t \in[0,1]^{S^{[\leqslant k]} \backslash \Sigma_{\tau}^{[\leqslant k]}}: f\left(y^{\prime}, t\right)>\lambda\right\}\right| \geqslant \varepsilon\right\} .
$$

For all $y=\left(y^{\prime}, t\right) \in[0,1]^{S[\leqslant k]}$, we let $f_{\varepsilon}(y):=\min \left(f(y), g_{f, \varepsilon}\left(y^{\prime}\right)\right)$ and

$$
A_{\varepsilon}:=\left\{y \in[0,1]^{S[\leqslant k]}: f(y)>g_{f, \varepsilon}\left(y^{\prime}\right)\right\}
$$

so that $f \geqslant f_{\varepsilon}$ and $f=f_{\varepsilon}$ on $[0,1]^{[\leqslant k]} \backslash A_{\varepsilon}$. From the definition of $g_{f, \varepsilon}$ and the equality

$$
\left|A_{\varepsilon}\right|=\int_{[0,1]^{\Sigma_{\tau}}[\leqslant k]}\left|\left\{t \in[0,1]^{S \leqslant k]} \backslash \Sigma_{\tau}^{[\leqslant k]}: f\left(y^{\prime}, t\right)>g_{f, \varepsilon}\left(y^{\prime}\right)\right\}\right| d y^{\prime}
$$

it follows that $\left|A_{\varepsilon}\right| \leqslant \varepsilon$. Moreover, the function $t \rightarrow f_{\varepsilon}\left(y^{\prime}, t\right)$ has essential supremum $g_{f, \varepsilon}\left(y^{\prime}\right)$, and attains such value on a set of measure bounded below by $\varepsilon$. We then get

$$
\begin{aligned}
& \left|\left\{y \in[0,1]^{S^{[\leqslant k]}}: f(y)>f\left(\tau_{0}^{*}(y), t\right) \widetilde{\forall} t \in[0,1]^{S^{[\leqslant k]} \backslash \Sigma_{\tau}^{[\leqslant k]}}\right\}\right| \\
& \leqslant\left|\left\{y \in[0,1]^{S[\leqslant k]} \backslash A_{\varepsilon}: f_{\varepsilon}(y)>f\left(\tau_{0}^{*}(y), t\right) \widetilde{\forall} t \in[0,1]^{S[\leqslant k] \backslash \Sigma_{\tau}^{[\leqslant k]}}\right\}\right|+\left|A_{\varepsilon}\right| \\
& \leqslant\left|\left\{y \in[0,1]^{S^{[\leqslant k]}} \backslash A_{\varepsilon}: f_{\varepsilon}(y)>f_{\varepsilon}\left(\tau_{0}^{*}(y), t\right) \widetilde{\forall} t \in[0,1]^{S^{[\leqslant k]} \backslash \Sigma_{\tau}^{[\leqslant k]}}\right\}\right|+\varepsilon \\
& \leqslant\left|\left\{y \in[0,1]^{S^{[\leqslant k]}}: f_{\varepsilon}(y)>f_{\varepsilon}\left(\tau_{0}^{*}(y), t\right) \widetilde{\forall} t \in[0,1]^{S^{[\leqslant k]} \backslash \Sigma_{\tau}^{[\leqslant k]}}\right\}\right|+\varepsilon \\
& \leqslant\left|\left\{y \in[0,1]^{S[\leqslant k]}: f_{\varepsilon}(y)>\operatorname{esssup}_{t \in[0,1]^{S[\leqslant k]} \backslash \Sigma_{\tau}^{[\leqslant k]}} f_{\varepsilon}\left(\tau_{0}^{*}(y), t\right)\right\}\right|+\varepsilon \\
& \leqslant\left|\left\{y \in[0,1]^{S \leqslant k]}: g_{f, \varepsilon}\left(\left.y\right|_{\Sigma_{\tau}^{[\leqslant k]}}\right)>g_{f, \varepsilon}\left(\tau_{0}^{*}(y)\right)\right\}\right|+\varepsilon \text {. }
\end{aligned}
$$


Since $\varepsilon$ was arbitrary, (4.10) implies that

$$
\begin{aligned}
& \sup _{f:[0,1]^{S[k k]} \rightarrow[0,1]}\left|\left\{y \in[0,1]^{S^{[\leqslant k]}}: f(y)>f\left(\tau_{0}^{*}(y), t\right) \widetilde{\forall} t \in[0,1]^{S^{[\leqslant k]} \backslash \Sigma_{\tau}^{[\leqslant k]}}\right\}\right| \\
& =\sup _{g:[0,1]^{\Sigma_{\tau}^{[\leqslant k]}} \rightarrow[0,1]}\left|\left\{y \in[0,1]^{S^{[\leqslant k]}}: g\left(\left.y\right|_{\Sigma_{\tau}^{[\leqslant k]}}\right)>g\left(\tau_{0}^{*}(y)\right)\right\}\right|
\end{aligned}
$$

as the $\geqslant$ inequality follows immediately by considering functions $f$ depending only on the variables in $[0,1]^{\Sigma_{\tau}^{[\leqslant k]}}$. Putting together (4.8) and (4.11) we then get

$$
\begin{aligned}
& \sup _{f:[0,1]^{S \leqslant k]} \rightarrow[0,1]}\left|\left\{x \in[0,1]^{\mathbb{N}[\leqslant k]}: f\left(\left.x\right|_{S[\leqslant k]}\right)>f\left(\tau^{*} \sigma^{*}(x)\right) \forall \sigma \in \mathcal{S}\right\}\right| \\
& \sup _{f:[0,1]^{S[\leqslant k]} \rightarrow[0,1]}\left|\left\{y \in[0,1]^{S[\leqslant k]}: f(y)>f\left(\tau_{0}^{*}(y), t\right) \widetilde{\forall} t \in[0,1]^{S^{[\leqslant k]} \backslash \Sigma_{\tau}^{[\leqslant k]}}\right\}\right| \\
& =\sup _{g:[0,1]^{\Sigma_{\tau}^{[\leqslant k]}} \rightarrow[0,1]}\left|\left\{y \in[0,1]^{S[\leqslant k]}: g\left(\left.y\right|_{\Sigma_{\tau}^{[\leqslant k]}}\right)>g\left(\tau_{0}^{*}(y)\right)\right\}\right| .
\end{aligned}
$$

Step 5. Notice that, if $g_{n} \rightarrow g$ almost everywhere on $[0,1]^{\Sigma_{\tau}^{[\leqslant k]}}$ as $n \rightarrow+\infty$, then

$$
\begin{aligned}
& \liminf _{n \rightarrow+\infty}\left|\left\{y \in[0,1]^{S[\leqslant k]}: g_{n}\left(\left.y\right|_{\Sigma_{\tau}^{[\leqslant k]}}\right)>g_{n}\left(\tau_{0}^{*}(y)\right)\right\}\right| \\
& \geqslant\left|\left\{y \in[0,1]^{S^{[\leqslant k]}}: g\left(\left.y\right|_{\Sigma_{\tau}^{[\leqslant k]}}\right)>g\left(\tau_{0}^{*}(y)\right)\right\}\right| .
\end{aligned}
$$

This implies that the supremum in (4.12) can be taken over a dense class of $g$ 's, or equivalently of $f$ 's, so that in particular the inequality in (4.7) is indeed an equality.

From (4.7) with the equality and from (4.12) we then have

$$
\sup _{m \in \mathcal{M}_{\mathrm{c}}\left(\omega_{1}^{\mathbb{N}[k]}\right)} \inf _{v \in \mathbb{N}^{[k]}} m\left(A_{v}\right)=\sup _{g:[0,1]^{\Sigma_{\tau}^{[\leqslant k]}} \rightarrow[0,1]}\left|\left\{y \in[0,1]^{S[k k]}: g\left(\left.y\right|_{\Sigma_{\tau}^{[\leqslant k]}}\right)>g\left(\tau_{0}^{*}(y)\right)\right\}\right|
$$

and by Lemma 4.1 we finally get

$$
\sup _{m \in \mathcal{M}_{\mathrm{c}}\left(\omega_{1}^{\mathbb{N}[k]}\right)} \inf _{v \in \mathbb{N}^{[k]}} m\left(A_{v}\right)=1-\frac{1}{w\left(\tau_{0}\right)}=1-\frac{1}{w\left(\left.\tau\right|_{\Sigma_{\tau}}\right)}=\lambda_{G}
$$

Step 6. To prove that last assertion it is enough to fix $\varepsilon>0$ and consider a contractible measure $m \in \mathcal{M}_{\mathrm{c}}\left(\omega_{1}^{\mathbb{N}[k]}\right)$ such that

$$
\inf _{v \in \mathbb{N}^{[k]}} m\left(A_{v}\right) \geqslant \lambda_{G}-\varepsilon
$$

The conclusion then follows by choosing $\Omega=\omega_{1}^{\mathbb{N}^{[k]}}$ as probability space, equipped with the measure $m$, and letting $X_{v}=A_{v}$. In this way, we define a random graph $X$ with no infinite paths and such that $m\left(A_{v}\right) \geqslant \lambda_{G}-\varepsilon$ for all $v \in \mathbb{N}^{[k]}$. 
Notice that, if $\tau$ is equivalent to $\tau^{\prime}$, then $w\left(\left.\tau\right|_{\Sigma_{\tau}}\right)=w\left(\left.\tau^{\prime}\right|_{\Sigma_{\tau}^{\prime}}\right)$, so that

$$
\tau \sim \tau^{\prime} \quad \Longrightarrow \quad \lambda_{G_{\tau}}=\lambda_{G_{\tau^{\prime}}} .
$$

When $G$ is not simple, reasoning as above we get the following weaker version of Theorem 4.5.

Proposition 4.6. Let $\mathcal{C} \subset \mathcal{E}_{k}$ and assume that $S_{\tau}=S$ for all $\tau \in \mathcal{C}$, for some $S \subset \mathbb{N}$. Letting $G=G_{\mathcal{C}}$ we have

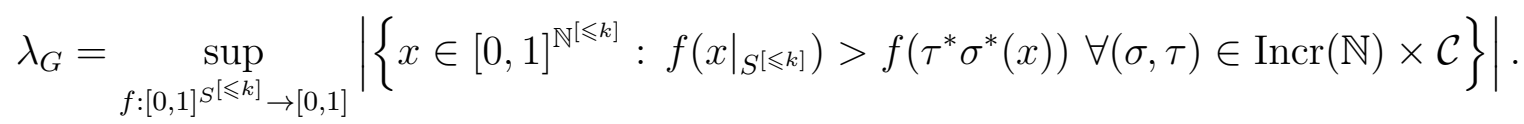

In particular, letting $X: \Omega \rightarrow 2^{V_{G}}$, the random graph $X$ has an infinite path if

$$
\lambda:=\inf _{v \in V_{G}} \mu\left(X_{v}\right)>\lambda_{G} .
$$

On the contrary, if $\lambda<\lambda_{G}$ we can find $X$ such that $X(\omega)$ has no infinite paths for some $\omega \in \Omega$.

The next result provides a sharp lower bound on the probability of having an infinite path.

Corollary 4.7. Assume that the sets $X_{v}$ are such that $\inf _{v \in V_{G}} \mu\left(X_{v}\right)=\lambda \geqslant \lambda_{G}$. Let $P_{\lambda}$ be the set of all $\omega \in \Omega$ such that $X(\omega)$ contains an infinite path. Then

$$
\mu\left(P_{\lambda}\right) \geqslant \frac{\lambda-\lambda_{G}}{1-\lambda_{G}}
$$

Proof. Consider the conditional probability $\mu\left(\cdot \mid \Omega \backslash P_{\lambda}\right) \in \mathcal{M}_{1}(\Omega)$. For all $v \in V_{G}$ we have

$$
\begin{aligned}
\mu\left(X_{v} \mid \Omega \backslash P_{\lambda}\right) & \geqslant \frac{\mu\left(X_{v}\right)-\mu\left(P_{\lambda}\right)}{1-\mu\left(P_{\lambda}\right)} \\
& \geqslant \frac{\lambda-\mu\left(P_{\lambda}\right)}{1-\mu\left(P_{\lambda}\right)} .
\end{aligned}
$$

Applying Theorem 4.5 or Proposition 4.6 to $\mu\left(\cdot \mid \Omega \backslash P_{\lambda}\right)$ it follows that $\frac{\lambda-\mu\left(P_{\lambda}\right)}{1-\mu\left(P_{\lambda}\right)} \leqslant \lambda_{G}$, or equivalently $\mu\left(P_{\lambda}\right) \geqslant \frac{\lambda-\lambda_{G}}{1-\lambda_{G}}$.

Remark 4.8. In the particular case of the graph $G_{k}$ defined in Section 3.3, we have $S_{\tau}=\{1, \ldots, k\}, \tau=\left.s\right|_{S_{\tau}}$ and $\Sigma_{\tau}=\{1, \ldots, k-1\}\left(\Sigma_{\tau}=\emptyset\right.$ if $\left.k=1\right)$. From Lemma 4.1 and Example 4.2 it then follows that the threshold $\lambda_{G_{k}}$ is given by

$$
\lambda_{G_{k}}=1-\frac{1}{k} .
$$

Moreover, by Theorem 4.5, $X<G_{k}$ contains an infinite path if $\lambda>\lambda_{G_{k}}$.

It is not clear from this analysis what happens when $\lambda=\lambda_{G_{k}}$, even if we expect that there are still infinite paths (this is proved in [FT:85] and [BMN:12] when $k=2$ ). 


\subsection{Paths of finite length}

Given a simple contractible graph $G=G_{\tau}$ and $p \in \mathbb{N}$, we can look for the threshold $\lambda_{p}$ such that the random graph $X$ contains a path of length $p$, whenever $\lambda>\lambda_{p}$. We can proceed exactly as in Theorem 4.5, with the simplification that the space $\omega_{1}^{\mathbb{N}^{[k]}}$ is replaced by $p^{\mathbb{N}^{[k]}}$, and obtain the following characterization of the threshold $\lambda_{p}$ :

$$
\lambda_{p}:=\sup \left\{\inf _{v \in V_{G}=\mathbb{N}^{[k]}} \mu\left(X_{v}\right): X \text { random graph without paths of length } p\right\} .
$$

Proposition 4.9. Let $p, k \in \mathbb{N}$. We have

$$
\begin{aligned}
\lambda_{p} & =\sup _{m \in \mathcal{M}_{1}\left(p^{\mathbb{N}[k]}\right)} \inf _{v \in \mathbb{N}^{[k]}} m\left(A_{v}\right) \\
& =\sup _{g:[0,1]^{\Sigma_{\tau}^{[\leqslant k]} \rightarrow p}}\left|\left\{y \in[0,1]^{S[\leqslant k]}: g\left(\left.y\right|_{\Sigma_{\tau}^{[\leqslant k]}}\right)>g\left(\left(\left.\tau\right|_{\Sigma_{\tau}^{[\leqslant k]}}\right)^{*}(y)\right)\right\}\right| .
\end{aligned}
$$

In particular, the random graph $X$ has a path of length $p$ if

$$
\inf _{v \in V_{G}} \mu\left(X_{v}\right)>\lambda_{p}
$$

On the other hand, if $\lambda<\lambda_{p}$ we can find $X$ and $\omega \in \Omega$ such that $X(\omega)$ has no paths of length $p$, and $\mu\left(X_{v}\right) \geqslant \lambda$ for all $v \in V_{G}$.

Notice that from Proposition 4.9 it follows that $\lambda_{p}$ is an increasing function of $p$ and $\lim _{p \rightarrow \infty} \lambda_{p}=\lambda_{G}$.

When $G=G_{k}$ we are able to explicitly compute the value of the threshold $\lambda_{p}$, which we denote by $\lambda_{p, k}$.

Proposition 4.10. Let $p, k \in \mathbb{N}$. Then

$$
\left(1-\frac{1}{k-1}\left\lceil\frac{k-1}{p}\right\rceil\right)\left(1-\frac{1}{k}\right) \leqslant \lambda_{p, k} \leqslant\left(1-\frac{1}{k-1}\left\lfloor\frac{k-1}{p}\right\rfloor\right)\left(1-\frac{1}{k}\right)
$$

In particular, if $(k-1)$ is a multiple of $p$ we get

$$
\lambda_{p, k}=\left(1-\frac{1}{p}\right)\left(1-\frac{1}{k}\right) \text {. }
$$

Proof. As in Example 4.2 we have $S=\{1, \ldots, k\}, \tau=\left.s\right|_{S}$ where $s$ is the shift map, and $\Sigma_{\tau}=\{1, \ldots, k-1\}$ (we set $\Sigma=\emptyset$ if $k=1$ ). Let us consider the function $h:[0,1]^{k-1} \rightarrow p$ defined as

$$
h\left(y_{1}, \ldots, y_{k-1}\right)=\bar{j}-1 \quad(\bmod p),
$$


where the index $\bar{j}$ is such that $y_{\bar{j}}=\max _{1 \leqslant i \leqslant k-1} y_{i}$, as in the proof of Lemma 4.1. We then have

$$
\begin{aligned}
\lambda_{p, k} & =\sup _{g:[0,1]_{\Sigma_{\tau}}^{[\leqslant k-1]} \rightarrow p}\left|\left(\left\{y \in[0,1]^{[\leqslant k-1]}: g\left(\left.y\right|_{\Sigma_{\tau}^{[\leqslant k-1]}}\right)>g\left(\left(\left.\tau\right|_{\Sigma_{\tau}}\right)^{*}(y)\right)\right\}\right)\right| \\
& =\sup _{g:[0,1]^{k-1} \rightarrow p}\left|\left\{y \in[0,1]^{k}: g\left(y_{1}, \ldots, y_{k-1}\right)>g\left(y_{2}, \ldots, y_{k}\right)\right\}\right| \\
& \geqslant\left|\left\{y \in[0,1]^{k}: h\left(y_{1}, \ldots, y_{k-1}\right)>h\left(y_{2}, \ldots, y_{k}\right)\right\}\right| \\
& \geqslant 1-\frac{1}{k}\left(1+\left\lceil\frac{k-1}{p}\right\rceil\right)=\left(1-\frac{1}{k-1}\left\lceil\frac{k-1}{p}\right\rceil\right)\left(1-\frac{1}{k}\right) .
\end{aligned}
$$

In order to prove the opposite inequality, for all functions $g:[0,1]^{\Sigma_{\tau}^{[\leqslant k-1]}} \rightarrow p$ we let

$$
A_{g}:=\left\{y \in[0,1]^{S^{[\leqslant k-1]}}: g\left(\left.y\right|_{\Sigma_{\tau}^{[\leqslant k-1]}}\right)>g\left(\left(\left.\tau\right|_{\Sigma_{\tau}}\right)^{*}(y)\right)\right\} \subset[0,1]^{[\leqslant k-1]} .
$$

Let $\tilde{\tau}: S=\{1, \ldots, k\} \rightarrow S$ be the $k$-periodic function defined as $\tilde{\tau}(i)=i+1$ if $i<k$, and $\tilde{\tau}(k)=1$. We have

$$
k\left(1-\left|A_{g}\right|\right)=k\left|A_{g}^{c}\right| \geqslant \int_{[0,1]^{S}[\leqslant k-1]} \sum_{j=0}^{k-1} \chi_{\left(\left(\tilde{\tau}^{*}\right)^{j} A_{g}\right)^{c}}(x) d x \geqslant\left\lceil\frac{k}{p}\right\rceil=1+\left\lfloor\frac{k-1}{p}\right\rfloor
$$

where $\chi_{A}$ denotes the characteristic function of a set $A \subset[0,1]^{S[k-1]}$. It then follows

$$
\left|A_{g}\right| \leqslant\left(1-\frac{1}{k-1}\left\lfloor\frac{k-1}{p}\right\rfloor\right)\left(1-\frac{1}{k}\right) .
$$

From Proposition 4.10, for all $k$ odd we get

$$
\lambda_{2, k}=\frac{k-1}{2 k}
$$

In particular $\lambda_{2,5}=2 / 5$, thus confirming a conjecture made in [TW:98]. We point out that in [TW:98] it is also shown that $\lambda_{2,6}>5 / 12$, so that (4.15) cannot hold for all couples $(p, k)$.

\subsection{Graphs with random edges}

In this final section we discuss a variant of Problem 1 for graphs with random edges. More precisely, given a graph $G$ with vertex set $\mathbb{N}^{[k]}$, we associate to each edge $e \in E_{G}$ a measurable set $X_{e} \subseteq \Omega$, where $(\Omega, \mu)$ is a given probability space. As before, the random graph can be equivalently defined by means of a $\mu$-measurable function $X: \Omega \rightarrow 2^{E_{G}}$, such that $X_{e}:=\{\omega \in \Omega: e \in X(\omega)\}$ for all $e \in E_{G}$. In this setting, Problem 1 becomes: 
Problem 2. For all $e \in E_{G}$ let $X_{e}$ be a measurable subset of $(\Omega, \mu)$, with $\mu\left(X_{e}\right) \geqslant \lambda \in$ $[0,1]$. We ask for which values of $\lambda$ there exists an infinite sequence of vertices $v_{i}$ of $G$ such that $\left(v_{i}, v_{i+1}\right) \in E_{G}$ for all $i \in \mathbb{N}$ and $\bigcap_{i \in \mathbb{N}} X_{v_{i}, v_{i+1}}$ is non-empty.

As above, answering to this question amounts to computing the threshold

$$
\widetilde{\lambda}_{G}:=\sup \left\{\inf _{e \in E_{G}} \mu\left(X_{e}\right): X \text { random subgraph of } G \text { without infinite paths }\right\} .
$$

Reasoning as in Section 4 , we let $X_{v}:=\cup_{v^{\prime}:\left(v, v^{\prime}\right) \in E_{G}} X_{v, v^{\prime}}, Y_{v} \subset X_{v}$ be the subset of all $\omega$ such that $X(\omega)$ contains an infinite path starting from $v$, and the map $\varphi: \Omega \rightarrow\left(\omega_{1}+1\right)^{\mathbb{N}^{[k]}}$ be defined as

$$
\varphi(\omega)_{v}= \begin{cases}\sup _{\left\{v^{\prime} \in \mathbb{N}^{[k]}:\left(v, v^{\prime}\right) \in E_{G}, \omega \in X_{v, v^{\prime}}\right\}} \varphi(\omega)_{v^{\prime}}+1 & \text { if } \omega \in X_{v} \backslash Y_{v}, \\ 0 & \text { if } \omega \notin X_{v} \\ \omega_{1} & \text { if } \omega \in Y_{v} .\end{cases}
$$

As before, if $X$ does not contain infinite paths then $\varphi: \Omega \rightarrow \omega_{1}^{\mathbb{N}^{[k]}}$ is essentially bounded and

$$
\varphi\left(X_{v, v^{\prime}}\right) \subset A_{v, v^{\prime}}:=\left\{x \in \omega_{1}^{\mathbb{N}^{[k]}}: x_{v}>x_{v^{\prime}}\right\} \quad \text { for all }\left(v, v^{\prime}\right) \in E_{G} .
$$

We now state the analog of Theorem 4.5 and Proposition 4.6 in this setting.

Theorem 4.11. Let $\mathcal{C} \subset \mathcal{E}_{k}$ and assume that $S_{\tau}=S$ for all $\tau \in \mathcal{C}$, for some $S \subset \mathbb{N}$. Letting $G=G_{\mathcal{C}}$ we have

$$
\begin{aligned}
\widetilde{\lambda}_{G} & =\sup _{m \in \mathcal{M}_{\mathrm{c}}\left(\omega_{1}^{\mathbb{N}[k]}\right)} \inf _{\left(v, v^{\prime}\right) \in E_{G}} m\left(A_{v, v^{\prime}}\right) \\
& =\sup _{f:[0,1]^{S[\leqslant k]} \rightarrow[0,1]}\left|\left\{x \in[0,1]^{\mathbb{N}[\leqslant k]}: f\left(\left.x\right|_{S[\leqslant k]}\right)>f\left(\tau^{*}(x)\right) \forall \tau \in \mathcal{C}\right\}\right| .
\end{aligned}
$$

When $G=G_{\tau}$ is a simple graph, from Lemma 4.1 it also follows

$$
\widetilde{\lambda}_{G}=1-\frac{1}{w(\tau)} .
$$

In particular, the random graph $X$ has an infinite path if

$$
\lambda:=\inf _{v \in V_{G}} \mu\left(X_{v}\right)>\widetilde{\lambda}_{G}
$$

On the other hand, if $\lambda<\widetilde{\lambda}_{G}$ we can find $X$ and $\omega \in \Omega$ such that $X(\omega)$ has no infinite paths and $\mu\left(X_{e}\right) \geqslant \lambda$ for all $e \in E_{G}$.

Notice that as before we have

$$
\tau \sim \tau^{\prime} \Longrightarrow w(\tau)=w\left(\tau^{\prime}\right) \quad \Longrightarrow \quad \tilde{\lambda}_{G_{\tau}}=\tilde{\lambda}_{G_{\tau^{\prime}}} .
$$

We also point out that the proof of Theorem 4.11 is analogous to the proof of Theorem 4.5, and in fact it is slightly shorter, due to the fact that the sets $A_{v, v^{\prime}}$ have a simpler definition than the corrsponding sets $A_{v}$. 


\section{References}

[A:08] T. Austin, On exchangeable random variables and the statistics of large graphs and hypergraphs, Probability Surveys 5 (2008), 80-145.

[BMN:12] A. Berarducci, P. Majer, M. Novaga, Infinite paths and cliques in random graphs, Fund. Math. 216 (2012), 163-191.

[DC:83] D. de Caen, A note on the probabilistic approach to Turán's problem, J. Combin. Theory Ser. B 34 (1983), 340-349.

[DF:30] B. De Finetti, Funzione caratteristica di un fenomeno aleatorio, Mem. Accad. Naz. Lincei. Cl. Sci. Fis. Mat. Natur. ser. 6 vol. 4 (1930).

[EH:64] P. Erdős, A. Hajnal, Some remarks on set theory. IX: Combinatorial problems in measure theory and set theory, Mich. Math. J. 11 (1964), 107-127.

[FT:85] D. H. Fremlin, M. Talagrand, Subgraphs of random graphs, Trans. Amer. Math. Soc. 291 (1985), 551-582.

[HS:55] E. Hewitt and J. Savage, Symmetric measures on cartesian products, Trans. Amer. Math. Soc. 80 (1955), 470-501.

[K:92] O. Kallenberg, Symmetries of random arrays and set-indexed processes, J. Theor. Probab. 5 (1992), 727-765.

[K:05] O. Kallenberg, Probabilistic Symmetries and Invariance Principles, SpringerVerlag, New York (2005).

[TW:98] W. T. Trotter, P. M. Winkler, Ramsey theory and sequences of random variables, Probability, Combinatorics and Computing 7 (1998), 221-238.

[VV:09] I.V. Voloshin, Introduction to Graph and Hypergraph Theory, Nova Science Publishers, Inc., New York (2009). 